\title{
Cloning of oxidosqualene cyclases from Maytenus ilicifolia for synthetic biology
}

\author{
Cristina C Barbosa ${ }^{1 *}$, Thaís B Alves ${ }^{2}$, Sandro R Valentini ${ }^{1}$, Cleslei F Zanelli ${ }^{1}$, Maysa Furlan ${ }^{2}$, Tatiana M Souza-Moreira ${ }^{1}$ \\ From 5th Congress of the Brazilian Biotechnology Society (SBBIOTEC) \\ Florianópolis, Brazil. 10-14 November 2013
}

\section{Introduction}

Pentacyclic triterpenes are secondary metabolites which are promising molecules in the pharmaceutical field and food and material industries. These compounds are formed by the cyclization of oxidosqualene catalyzed by oxidosqualene cyclases (OSC) [1]. The aim of this study is to clone the OSC genes from the leaves of Maytenus illicifolia, a medicinal species from Brazil.

\section{Material and methods}

For this purpose, we adopted the strategy of RT-PCR using degenerated primers obtained from sequences of known plant OSC genes. Total RNA was extracted from leaves of M. ilicifolia and used in the synthesis of cDNA. The PCR amplification of core fragments of OSC genes was performed with partially degenerated primers designed to anneal to highly conserved regions among OSC genes. The cloned fragments were sequenced and specific primers were designed for the rapid amplification of cDNA end (RACE) of 3' regions and for a first round amplification of 5 ' extension. After sequencing, another set of specific primers were generated for the RACE of 5' regions. Finally, it was possible to clone the full-length cDNA of the OSCs and confirm the predicted identity of the genes by sequencing [2].

\section{Results and discussion}

In silico assembly and sequencing of the full-length cDNA covered the ORF of two main different groups of OSCs with $\sim 2500 \mathrm{bp}$. Multiple alignment of these genes showed identity of about $50 \%$ between those two groups. Comparison of $M$. ilicifoliaOSC genes with those described in the Genebank revealed that one group showed high identity $(\sim 90 \%)$ with cycloartenol synthase enzymes, while the other one showed high identity $(>75 \%)$ with triterpene synthases as beta-amyrin and lupeol synthases.

\section{Conclusions}

Cloning of OSC genes from the leaves of M. ilicifolia demonstrated two main groups of OSC enzymes present: the cycloartenol synthase, which is part of the plant primary metabolism and one triterpene synthase, which may be part of the secondary metabolism [3]. Future work using functional expression of these cloned genes in Saccharomyces cerevisiae will further characterize the oxidosqualene cyclases from M. ilicifolia leaves.

\section{Acknowledgements \\ PADC-UNESP, CNPq andFAPESP}

\section{Authors' details}

${ }^{1}$ Faculdade de Ciências Farmacêuticas, UNESP, Sao Paulo State University, SP Brazil. ${ }^{2}$ Instituto de Química, UNESP, Sao Paulo State University, SP, Brazil.

Published: 1 October 2014

\section{References}

1. Withers $\mathrm{S}$, Keasling J: Biosynthesis and engineering of isoprenoid small molecules. Appl Microbiol Biotechnol 2007, 73:980-990.

2. Wang Z, Yeats $T$, Han $H$, Jetter R: Cloning and characterization of oxidosqualene cyclases from Kalanchoe daigremontiana. J Biol Chem 2010, 285:29703-29712.

3. Phillips DR, Rasbery JM, Bartel B, Matsuda SPT: Biosynthetic diversity in plant triterpene cyclization. Curr Opin Plant Biol 2006, 9:305-314.

doi:10.1186/1753-6561-8-S4-P248

Cite this article as: Barbosa et al:: Cloning of oxidosqualene cyclases from Maytenus ilicifolia for synthetic biology. BMC Proceedings 2014 8(Suppl 4):P248. 